\section{Does Measuring Serum Concentration of Procalcitonin in Critically III Patients Assist in Stopping Antibiotic Therapy?}

\section{THE "PRO" SIDE}

Most successful debates start with a good understanding of the question at hand. We would like to begin the "pro" side of this debate by examining the question in detail. Like most diagnostic tests, the procalcitonin (PCT) test itself serves little purpose if not acted upon. In other words, for PCT monitoring to be helpful, it must possess characteristics that make it actionable. In addition, for the purpose of this debate, we will consider critically ill patients to be those who are treated in the intensive care unit and will ignore use of PCT measurement in the emergency department or other inpatient or outpatient settings. Lastly, reference to "stopping antibiotic therapy" signifies that an antibiotic has already been started. Simply put, the initial decision to start antibiotics has passed (independent of any quantification of PCT), and the decision now is whether the antibiotics can be de-escalated or discontinued. Such a change might occur because new clinical information and/or the PCT result has rendered obsolete the original decision to start antibiotics, or because the original infection has been adequately treated. In the remainder of this article, we will discuss in more detail the use of PCT measurement within the context of this question and provide recommendations on its most appropriate use. .

\section{Is PCT Actionable?}

More than a decade ago, PCT was deemed to be a "SMART" biomarker (specific and sensitive, measurable with precision, available and affordable, responsive and reproducible, and timely). ${ }^{1}$ Today, PCT remains a SMART biomarker, with newer amplified cryptate emission assays having even greater sensitivity. The PCT concentration in serum is normally below $0.1 \mu \mathrm{g} / \mathrm{L}$, but rises substantially in response to bacterial infections, endotoxins, and inflammatory cytokine. ${ }^{1}$ Because the concentration peaks between 8 and $24 \mathrm{~h}$ after an insult and because PCT has an elimination half-life between 22 and $35 \mathrm{~h}$, determining PCT concentration strikes the balance between having a measurable clinical window and ensuring responsiveness to treatments and disease progression. ${ }^{1}$ At a cost of about Can $\$ 50$ per assay, PCT measurement is less expensive than the daily charges for most antibiotics, ${ }^{2}$ and is therefore a potentially cost-effective antimicrobial stewardship tool. For centres that have the platform to run PCT assays, the results can be readily available and actionable within several hours of sampling.

Many different PCT-guided treatment algorithms for stopping antibiotics exist. In a recent study by de Jong and colleagues, ${ }^{3}$ patients admitted to the intensive care unit (ICU) with initiation of empiric antibiotics were randomly assigned to either PCT-guided treatment or usual care. For those in the PCT guidance group, PCT levels were obtained at baseline (within $24 \mathrm{~h}$ of antibiotic initiation) and daily until discharge from the ICU or 3 days after systemic antibiotics were stopped. The study protocol recommended that antibiotics be stopped if PCT concentration decreased by more than $80 \%$ from baseline or when the absolute value was less than $0.5 \mu \mathrm{g} / \mathrm{L}$. This algorithm is straightforward and led to significant decreases in the duration of antibiotic therapy and also mortality at 28 days. ${ }^{3}$

\section{Does PCT Monitoring Help in Stopping Antibiotics?}

The short answer to the question in this heading is "yes"! At least 8 meta-analyses specifically evaluating critically ill patients have concluded that utilization of PCT monitoring is associated with shorter duration of antimicrobial therapy. ${ }^{2,4-10}$ However, there are certain scenarios in which reliance on PCT values is not ideal. For example, use of elevated PCT level to justify initiation of antimicrobials or escalation of the antimicrobial spectrum of activity leads to overuse of antibiotics and potentially more end-organ dysfunction. ${ }^{11,12}$ Hence, using PCT as a screening tool to initiate antibiotics or to broaden antibiotic coverage in the absence of signs and symptoms of infection cannot be recommended. Furthermore, in the initiation phase of antimicrobials, clinicians may not be inclined to withhold initial antibiotics. This was evident in a study by Layios and others, ${ }^{12}$ in which samples for measurement of PCT were drawn when clinicians suspected infection. In that study, for cases in which baseline PCT was normal, only $36 \%$ of physicians were compliant with withholding antibiotics; in contrast, for patients with elevated baseline PCT, $86 \%$ of clinicians were compliant with continuing antibiotics. Hence, sampling PCT in the initiation phase is unlikely to overrule clinical judgment regarding antibiotics and may be more useful in the de-escalation phase of antimicrobial management. In fact, a recent meta-analysis specifically evaluated studies of PCT during the de-escalation phase, and concluded that both duration of antibiotic therapy (days) and short-term mortality were 
significantly lower with PCT guidance when used for deescalation purposes, but not when used for initiation of therapy or a mixture of the 2 approaches. ${ }^{4}$

\section{How Should PCT Be Used?}

No biomarker should be used in isolation for decisionmaking. Among critically ill patients, PCT monitoring should be part of a comprehensive antimicrobial stewardship program, with clinician guidance on how to interpret the test results. If the decision is made to initiate antibiotics and it is anticipated that PCT will be used for cessation decision-making, sampling for PCT should be completed at baseline purely for trending purposes. PCT level can be checked again on day 3 if clinicians are questioning the initial decision to initiate antibiotics (i.e., whether antibiotics should be continued). Beyond day 3, for patients whose condition is improving, PCT can be checked to determine whether the infection has been adequately treated. In both scenarios, the algorithm described by de Jong and others $^{3}$ is a reasonable approach, with recommendations to stop or de-escalate antibiotics when the absolute value of PCT is less than $0.5 \mu \mathrm{g} / \mathrm{L}$ or the PCT value has decreased at least $80 \%$ from baseline. When used for the de-escalation of antibiotics, PCT is consistently associated with reductions in antibiotic usage and may improve short-term mortality.

\section{References}

1. Shehabi Y, Seppelt I. Pro/Con debate: Is procalcitonin useful for guiding antibiotic decision making in critically ill patients? Crit Care. 2008;12(3):211.

2. Heyland DK, Johnson AP, Reynolds SC, Muscedere J. Procalcitonin for reduced antibiotic exposure in the critical care setting: a systematic review and an economic evaluation. Crit Care Med. 2011;39(7):1792-9.

3. de Jong E, van Oers JA, Beishuizen A, Vos P, Vermeijden WJ, Haas LE, et al. Efficacy and safety of procalcitonin guidance in reducing the duration of antibiotic treatment in critically ill patients: a randomised, controlled, open-label trial. Lancet Infect Dis. 2016;16(7):819-27.

4. Lam SW, Bauer SR, Fowler R, Duggal A. Systematic review and meta-analysis of procalcitonin-guidance versus usual care for antimicrobial management in critically ill patients: focus on subgroups based on antibiotic initiation, cessation, or mixed strategies. Crit Care Med. 2018;46(5):684-90.

5. Agarwal R, Schwartz DN. Procalcitonin to guide duration of antimicrobial therapy in intensive care units: a systematic review. Clin Infect Dis. 2011; 53(4):379-87.

6. Andriolo BN, Andriolo RB, Salomão R, Atallah ÁN. Effectiveness and safety of procalcitonin evaluation for reducing mortality in adults with sepsis, severe sepsis or septic shock. Cochrane Database Syst Rev. 2017;1:CD010959.

7. Kopterides P, Siempos, II, Tsangaris I, Tsantes A, Armaganidis A. Procalcitonin-guided algorithms of antibiotic therapy in the intensive care unit: a systematic review and meta-analysis of randomized controlled trials. Crit Care Med. 2010;38(11):2229-41.

8. Matthaiou DK, Ntani G, Kontogiorgi M, Poulakou G, Armaganidis A, Dimopoulos G. An ESICM systematic review and meta-analysis of procalcitonin-guided antibiotic therapy algorithms in adult critically ill patients. Intensive Care Med. 2012;38(6):940-9.

9. Prkno A, Wacker C, Brunkhorst FM, Schlattmann P. Procalcitonin-guided therapy in intensive care unit patients with severe sepsis and septic shocka systematic review and meta-analysis. Crit Care. 2013;17(6):R291.

10. Soni NJ, Samson DJ, Galaydick JL, Vats V, Huang ES, Aronson N, et al. Procalcitonin-guided antibiotic therapy: a systematic review and metaanalysis. J Hosp Med. 2013;8(9):530-40
11. Jensen JU, Hein L, Lundgren B, Bestle MH, Mohr TT, Andersen MH, et al. Procalcitonin-guided interventions against infections to increase early appropriate antibiotics and improve survival in the intensive care unit: a randomized trial. Crit Care Med. 2011;39(9):2048-58.

12. Layios N, Lambermont B, Canivet JL, Morimont P, Preiser JC, Garweg C, et al. Procalcitonin usefulness for the initiation of antibiotic treatment in intensive care unit patients. Crit Care Med. 2012;40(8):2304-9.

Simon W Lam, PharmD, MS

Seth R Bauer, PharmD

Department of Pharmacy

Cleveland Clinic

Cleveland, Ohio

Competing interests: None declared.

\section{THE "CON" SIDE}

Procalcitonin (PCT) has grown in popularity recently as a surrogate marker for bacterial infection in patients with sepsis. After all, who would not want a test to guide the decision to stop or continue antibiotics in complex critically ill patients? The US Food and Drug Administration (FDA) approved PCT monitoring in 2017 to aid in the discontinuation of antibiotics in sepsis. ${ }^{1}$ Despite this approval, the role of PCT monitoring has been under continued passionate debate. The mixed results of multiple meta-analyses assessing the outcomes of PCT monitoring are reflected in a weak recommendation from the Infectious Diseases Society of America, which lists serial PCT measurement as a tool to support discontinuation of antibiotics. ${ }^{2}$ This situation has led to doubt about the preference for a surrogate marker over clinical judgment in critically ill patients.

The only predictable characteristic of PCT is its pharmacokinetics. Although it is believed that PCT can differentiate between bacterial and viral causes of systemic infection, a recent publication casts doubt. ${ }^{3}$ In that study, higher PCT levels were correlated with the increased probability of a bacterial infection, but no PCT threshold was identified that reliably distinguished between a bacterial and a viral infection. ${ }^{3}$ Overall, PCT is closely associated with, but is not specific for, infection; in essence, then, PCT measurement is similar to determining white blood cell concentration, but at much greater cost. Similar to the white blood cell count, PCT can be falsely elevated in patients with cardiogenic shock or a systemic inflammatory response to surgery, trauma, or burns. Falsely low values may be seen in localized infections and subacute endocarditis, or may occur if PCT is measured too early in the course of a systemic infection. ${ }^{4}$ These limitations may affect the utility of PCT measurement in critically ill patients, whose antibiotic therapy may be started or stopped inappropriately.

Death is the most important outcome of any diagnostic or therapeutic strategy, but multiple meta-analyses of PCT monitoring 
have reported mixed outcomes regarding the mortality benefit, depending on the design of the meta-analysis, the patient population assessed, and the role of PCT (for initiation or discontinuation of antibiotics, or both). This topic has been so popular that 5 metaanalyses of patients with sepsis in the intensive care unit (ICU) were published in 2017 and 2018..$^{5-9}$ Andriolo and others ${ }^{5}$ limited their assessment to trials using strict definitions of sepsis, severe sepsis, or septic shock. They found no mortality difference at 28 days (risk ratio [RR] $0.89,95 \%$ confidence interval [CI] 0.61-1.31) or at longest follow-up (RR 0.81, 95\% CI 0.65-1.01), not even when less stringent trial criteria were used as part of the sensitivity analysis. ${ }^{5}$ Two meta-analyses limited their analysis to trials assessing PCT evaluation for the purpose of cessation of antibiotics in critically ill patients. In both, there was a decrease in mortality: RR $0.86(95 \%$ CI 0.76-0.98) ${ }^{6}$ and RR 0.87 (95\% CI 0.77-0.98). ${ }^{7}$ The decrease in mortality may be due to their inclusion of a large randomized controlled trial (RCT) by de Jong and others. ${ }^{10}$ Of the numerous RCTs evaluating PCT, this was the only RCT to show a mortality benefit (absolute difference 6.6\%, 95\% CI 1.3\%-11.9\%). The authors proposed that this benefit might be due to earlier identification of an alternative diagnosis..$^{10}$ However, the same reduced mortality was not seen in a meta-analysis created as part of a regulatory submission to the FDA, ${ }^{8}$ despite inclusion of the de Jong trial. That meta-analysis assessed both patient- and study-level data, but did not show a reduction in mortality for either. ${ }^{8}$ In a subsequent metaanalysis, Wirz and others ${ }^{9}$ used only patient-level data and found a reduction in mortality (adjusted odds ratio 0.89 , 95\% CI 0.8-0.99). The bottom line is that most of these meta-analyses were analyzing the same RCTs, with outcomes that varied depending on how the analyses were structured. From these discordant mortality results, it is difficult to understand the true effect of the intervention. At most, we can say that the PCT-guided therapy did no worse than standard of care. However, given that there have been more meta-analyses published recently on this topic than RCTs, we must ask whether the answer is hidden in the data, or are we simply looking for an effect where none exists?

If a reduction in mortality cannot be found, then what about other outcomes? The biggest push for PCT monitoring has been based on reducing exposure to antibiotics as a means of reducing the potential for antimicrobial resistance. The meta-analyses cited above, regardless of design, all showed a decrease in duration of antibiotic use (by 1 or 2 days).-9 These results are not surprising, given the open-label trial designs and close assessment of patients in the intervention arms. Despite the positive results from individual RCTs and meta-analyses, real-world outcomes have been suboptimal. In a retrospective cohort study of 107 ICUs in the United States with PCT monitoring available, PCT-guided therapy led to an increase in antibiotic use (adjusted relative risk 1.1, 95\% CI 1.15-1.18) with no difference in mortality (hazard ratio 1.05, 95\% CI $0.93-1.19) .{ }^{11}$ These results are concerning because of potential increases in costs and adverse effects.

If we just ignore the conflicting data and assume that the benefits of PCT monitoring outweigh its risks, one question remains: Will clinicians use PCT monitoring in their practice? The retrospective ICU cohort showed that PCT monitoring was ordered for only $18 \%$ of patients upon initiation of antibiotics for sepsis, and only $29.4 \%$ of these patients underwent subsequent measurement of PCT level. ${ }^{11}$ Clinicians participating in RCTs were only slightly better in terms of their adherence to protocols: when compliance was measured, it was generally poor. ${ }^{10,12-14}$ Even in the largest trial, almost half of the clinicians chose to overrule the discontinuation guidelines unless the patient was clinically stable. ${ }^{10}$ Perhaps noncompliance is due to the lack of validation of clinical decision algorithms for use of PCT monitoring to discontinue antibiotics in sepsis. PCT thresholds in sepsis trials have been heterogeneous with respect to cut-off values, percent changes from baseline, and monitoring strategies. Before PCT monitoring can be confidently implemented and interpreted by clinicians, further studies are needed to prospectively validate cut-off thresholds. After all, why order a test if you do not know what to do with the results?

Although there is no perfect biomarker for sepsis, it is unclear what value monitoring PCT adds to clinical judgment that would offset the cost of this expensive test. It has the potential for overutilization and increased costs, and is limited by inadequate clinician response or acceptance of results. The proposed mortality benefit is cause for excitement, but should be interpreted cautiously, given that it has been observed in only one clinical trial. Overall, although the intentions of using PCT monitoring are generally favourable, the evidence has remained controversial and the jury is still out on the benefits. Next time you want to order a PCT monitoring test ... just look at the white blood cell count instead and use your clinical judgment.

\section{References}

1. FDA clears test to help manage antibiotic treatment for lower respiratory tract infections and sepsis [news release]. Silver Spring (MD): US Food and Drug Administration; 2017 [cited 2018 Aug 22]. Available from: https:// www.fda.gov/newsevents/newsroom/pressannouncements/ucm543160.htm

2. Barlam TF, Cosgrove SE, Abbo LM, MacDougall C, Schuetz AN, Septimus EJ, et al. Implementing an antibiotic stewardship program: guidelines by the Infectious Diseases Society of America and the Society for Healthcare Epidemiology of America. Clin Infect Dis. 2016;62(10):e51-77.

3. Self WH, Balk RA, Grijalva CG, Williams DJ, Zhu Y, Anderson EJ, et al. Procalcitonin as a marker of etiology in adults hospitalized with communityacquired pneumonia. Clin Infect Dis. 2017;65(2):183-90.

4. Becker KL, Snider R, Nylen ES. Procalcitonin assay in systemic inflammation, infection, and sepsis: clinical utility and limitations. Crit Care Med. 2008; 36(3):941-52.

5. Andriolo BN, Andriolo RB, Salomão R, Atallah ÁN. Effectiveness and safety of procalcitonin evaluation for reducing mortality in adults with sepsis, severe sepsis or septic shock. Cochrane Database Syst Rev. 2017;1:CD010959.

6. Huang H, Peng JM, Weng L, Wang CY, Jiang W, Du B. Procalcitoninguided antibiotic therapy in intensive care unit patients: a systematic review and meta-analysis. Ann Intensive Care. 2017;7(1):114.

7. Lam SW, Bauer SR, Fowler R, Duggal A. Systematic review and meta-analysis of procalcitonin-guidance versus usual care for antimicrobial management in critically ill patients: focus on subgroups based on antibiotic initiation, cessation, or mixed strategies. Crit Care Med. 2018;46(5):684-90.

8. Iankova I, Thompson-Leduc P, Kirson NY, Rice B, Hey J, Krause A, et al. Efficacy and safety of procalcitonin guidance in patients with suspected or confirmed sepsis: a systematic review and meta-analysis. Crit Care Med. 2018;46(5):691-8.

9. Wirz Y, Meier MA, Bouadma L, Luyt CE, Wolff M, Chastre J, et al. Effect of procalcitonin-guided antibiotic treatment on clinical outcomes in intensive 
care unit patients with infection and sepsis patients: a patient-level metaanalysis of randomized trials. Crit Care. 2018;22(1):191.

10. de Jong E, van Oers JA, Beishuizen A, Vos P, Vermeijden WJ, Haas LE, et al. Efficacy and safety of procalcitonin guidance in reducing the duration of antibiotic treatment in critically ill patients: a randomised, controlled, open-label trial. Lancet Infect Dis. 2016;16(7):819-27.

11. Chu DC, Mehta AB, Walkey AJ. Practice patterns and outcomes associated with procalcitonin use in critically ill patients with sepsis. Clin Infect Dis. 2017;64(11):1509-15.

12. Bouadma L. Use of procalcitonin to reduce patients' exposure to antibiotics in intensive care units (PRORATA trial): a multicentre randomised controlled trial. Lancet. 2010;375(9713):463-74.

13. Bloos F, Trips E, Nierhaus A, Briegel J, Heyland DK, Jaschinski U, et al. Effect of sodium selenite administration and procalcitonin-guided therapy on mortality in patients with severe sepsis or septic shock: a randomized clinical trial. JAMA Intern Med. 2016;176(9):1266-76.
14. Layios N, Lambermont B, Canivet JL, Morimont P, Preiser JC, Garweg C, et al. Procalcitonin usefulness for the initiation of antibiotic treatment in intensive care unit patients. Crit Care Med. 2012;40(8):2304-9.

Michelle Hinch, BScPharm, PharmD

Clinical Pharmacy Specialist, Antimicrobial Stewardship

St Paul's Hospital

Vancouver, British Columbia

Competing interests: None declared.

\section{ON THE FRONT COVER}

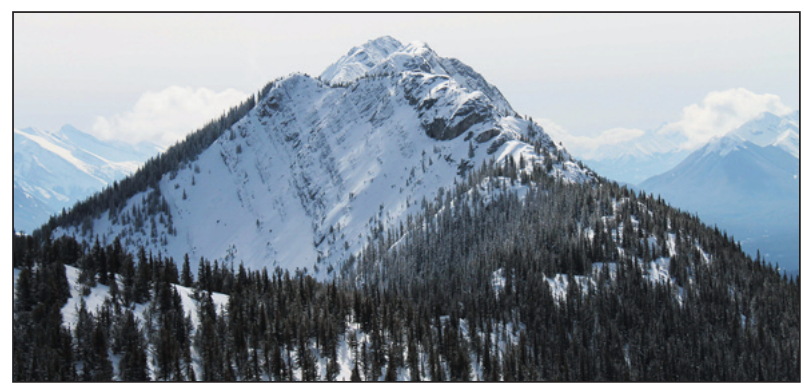

The CJHP would be pleased to consider photographs featuring Canadian scenery taken by CSHP members for use on the front cover of the Journal. If you would like to submit a photograph, please send

\section{Banff, Alberta}

Grace Lin took the cover photo while attending the CSHP Banff Seminar in March 2012. "Looking into the mountain ridges with the melting snow brought joy to my heart on a beautiful spring day." Grace captured the image with a Canon Rebel XS, her first digital DSLR camera. In addition to photography, Grace also dabbles in painting as a hobby. She has been practising as a hospital pharmacist since 2007 and is currently employed with Lions Gate Hospital in Vancouver.

an electronic copy (minimum resolution $300 \mathrm{dpi}$ ) to publications@cshp.pharmacy. 